\title{
Simulation of supersymmetric quantum mechanics in a Cooper-pair box shunted by a Josephson rhombus
}

\author{
Jascha Ulrich, Daniel Otten, and Fabian Hassler \\ JARA-Institute for Quantum Information, RWTH Aachen, D-52074, Germany
}

(Dated: November 2015)

\begin{abstract}
Supersymmetries in quantum mechanics offer a way to obtain degeneracies in the excitation spectrum which do not originate from selection rules. The mechanism behind the degeneracies is the same as the one that leads to the miraculous cancellations of divergences in supersymmetric field theories found in the high energy physics context. There is up to now no realistic proposal of nonintegrable systems that show level degeneracies due to a supersymmetric structure. Here, we propose an implementation of a quantum-mechanical supersymmetry in a Cooper-pair box shunted by a Josephson junction rhombus which is effectively $\pi$-periodic in the superconducting phase difference. For a characteristic ratio between the strength of the $2 \pi$ - and the $\pi$-periodic junction, we find a two-fold degeneracy of all the energy levels all the way from the weak junction/charge qubit limit to the strong junction/transmon regime. We provide explicit experimental values for the parameters of the system and show that tuning in and out of the supersymmetric point is easily achieved by varying an external gate voltage. We furthermore discuss a microwave experiment to detect the supersymmetry and conclude that it can indeed be implemented with currently existing Josephson junction technology.
\end{abstract}

PACS numbers: 03.67.Ac, 11.30.Pb, 85.25.Cp, 42.50.Pq

The macroscopic quantum mechanics of superconducting circuits has allowed the experimental simulation of many complex quantum phenomena such as phase transitions, ${ }^{1}$ quantum spins, ${ }^{2}$ or dynamics in open systems. ${ }^{3}$ Theoretically, the quantum simulation of intricate subjects such as Hawking radiation ${ }^{4}$ and lattice gauge theories ${ }^{5-7}$ has been proposed. In the plethora of phenomena that can be simulated with the help of superconducting circuits, ${ }^{8,9}$ degeneracies due to quantummechanical supersymmetries have notably been absent. Typically, degeneracies in the spectrum arise when the Hamiltonian commutes with all group elements of a nonAbelian symmetry which translates into selection rules demanding vanishing off-diagonal and equal diagonal matrix elements of the Hamiltonian within the same irreducible representation. ${ }^{10}$ The degeneracy of the states thus always follows from the dimension of the representation. Supersymmetry on the other hand does not simply forbid different states to couple but it makes sure that in each order of perturbation theory there is always a pair of terms canceling each other. ${ }^{11,12}$

It is intriguing that the degeneracies of supersymmetric quantum mechanics occur by the same mechanism ${ }^{11}$ that leads to a miraculous cancellation of divergences in supersymmetric field theories and makes supersymmetries an important tool of particle physics. ${ }^{13}$ In trivial cases like the free particle ${ }^{14}$ or the Jaynes-Cummings model, ${ }^{15}$ however, the supersymmetric structure is irrelevant since the spectrum is exactly solvable. In order to deepen the connection to the ideas in the high-energy context, it is thus of vital importance to propose a non-integrable system where the level degeneracy can be solely understood by its supersymmetric structure.

In this paper, we show that shunting a Cooper-pair box with a Josephson junction rhombus simulates a

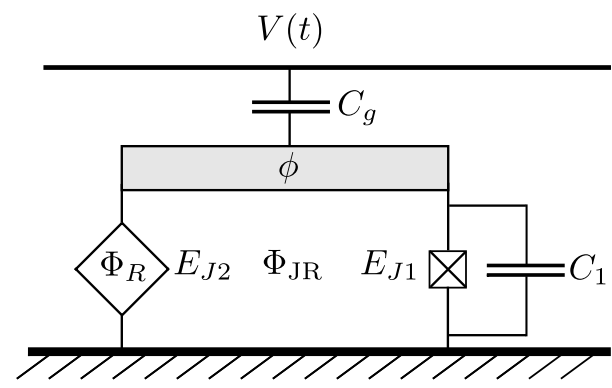

FIG. 1. Setup simulating the Hamiltonian Eq. (1). A Josephson junction (crossed box) with Josephson energy $E_{J 1}$ and capacitance $C_{1}$ couples the Cooper-pair box, a superconducting island with superconducting phase $\phi$, to a ground superconductor (diagonally striped) with phase $\phi_{0}=0$. A Josephson rhombus (depicted as a rhombus) provides an additional shunt to the ground which generates a $\cos (2 \phi)$ Josephson coupling of strength $E_{J 2}$ when the rhombus is threaded by a flux $\Phi_{R}=\Phi_{0} / 2$. We assume that an additional flux $\Phi_{J R}=-\Phi_{R} / 2$ threads the loop between the standard junction and the rhombus, which requires a second flux line that can be controlled separately. A capacitance $C_{g}$ couples the system to a transmission line at the voltage $V(t)$.

highly nontrivial supersymmetry that can be experimentally realized with today's Josephson junction technology. This proposal combines ideas of implementing supersymmetry in purely bosonic systems ${ }^{16}$ with the quantummechanical supersymmetry that has recently been proposed for superconductors hosting fermionic Majorana bound states. ${ }^{12}$ The only nonstandard component of our proposal is the Josephson rhombus. The Josephson rhombus is a junction between two superconductors that allows only tunneling of pairs of Cooper pairs. Consequently, its current-phase relation is $\pi$-periodic. ${ }^{17}$ 
Josehson rhombi have previously been proposed as building blocks for topologically protected qubits ${ }^{18,19}$ that have been shown to be experimentally feasible. ${ }^{20} \mathrm{Ad}-$ ditionally, they have been employed for the experimental realization of qubits based on the Cooper-pair parity. ${ }^{21} \mathrm{Up}$ to now, theoretical studies on the Josephson rhombi have mainly been focused on the semi-classical regime. ${ }^{22,23}$ For our setup, we study a Josephson rhombus in the charging limit where it is adiabatically coupled to the superconducting island and generates a $\pi$-periodic Josephson coupling of a specific $\cos (2 \phi)$ form. A supersymmetry is then obtained for a characteristic ratio between the strength of the conventional and the $\pi$-periodic Josephson junction, see below.

Our system of interest is depicted in Fig. 1. It is an extension of the conventional Cooper-pair box,${ }^{24,25}$ which consists of a superconducting island with superconducting phase $\phi$ and a ground superconductor with phase $\phi_{0}=0$ which are coupled by a Josephson junction with Josephson energy $E_{J 1}$ and capacitance $C_{1}$. A capacitance $C_{g}$ couples the system to a transmission line biased at a DC voltage $V(t)=V_{g}$. We add an additional shunt to the ground through a Josephson rhombus with capacitance $C_{\diamond}$, which, as we will discuss in more detail below, generates a $\pi$-periodic Josephson energy $-E_{J 2} \cos (2 \phi)$ when threaded by a flux $\Phi_{R}=\Phi_{0} / 2$, where $\Phi_{0}=h / 2 e$ is the superconducting flux quantum and $E_{J 2}$ the effective junction energy. Taking into account an additional flux $\Phi_{J R}=-\Phi_{R} / 2$ in the loop between the conventional junction and the rhombus, we obtain the effective lowenergy Hamiltonian

$$
H_{\mathrm{eff}}=4 E_{C_{\Sigma}}\left(n-n_{g}\right)^{2}-E_{J 1} \cos \phi-E_{J 2} \cos (2 \phi),
$$

where $n=-i \partial / \partial \phi$ is the number of Cooper pairs on the island, $E_{C_{\Sigma}}=e^{2} / 2 C_{\Sigma}$ with $C_{\Sigma}=C_{1}+C_{g}+C_{\diamond}$ is the total charging energy of the island, and $n_{g}=C_{g} V_{g} / 2 e$ is the induced offset charge in units of $2 e$. The Hamiltonian (1) does not admit an analytic solution. Its only symmetry is the operation $K: \phi \mapsto-\phi$ at the point $n_{g}=0$ which due to its Abelian nature does not lead to any degeneracy. However, as we will show below, for a specific ratio of the energy scales all excited levels are degenerate due to a supersymmetry.

In the simplest setting, a Hamiltonian $H_{Q}$ is called supersymmetric when there exists a Hermitian involution $K$ with $K^{2}=1$ that commutes with $H_{Q}$ and a Hermitian supercharge $Q$ which anticommutes with $K$ and factorizes the Hamiltonian $H_{Q}=Q^{2}{ }^{26,27}$ The sectors of $H_{Q}$ are then characterized by $K$ according to $H_{Q}=P_{+} H_{Q} P_{+}+P_{-} H_{Q} P_{-}$with $P_{ \pm}=(1 \pm K) / 2$ and are intertwined through the relation $P_{ \pm} Q=Q P_{\mp}$ which guarantees that to each eigenstate $|a\rangle$ to energy $E_{a}>0$ in one sector there is a partner state $\left(Q / \sqrt{E_{a}}\right)|a\rangle$ to the same energy in the other sector. To see how this relates to our system, let us introduce the supercharge $Q$ and the involution $K$ according to ${ }^{12}$

$$
Q=2 \sqrt{E_{C_{\Sigma}}}(n+i \alpha \sin \phi)(-1)^{n}, \quad K \phi K=-\phi,
$$

$(a)$

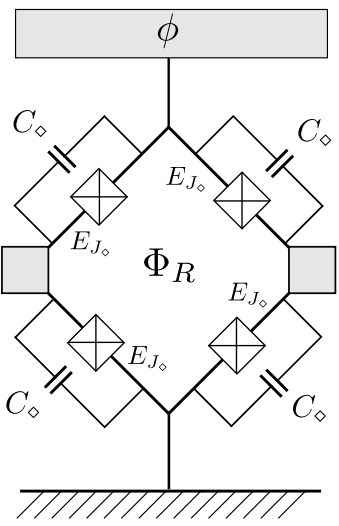

(b)

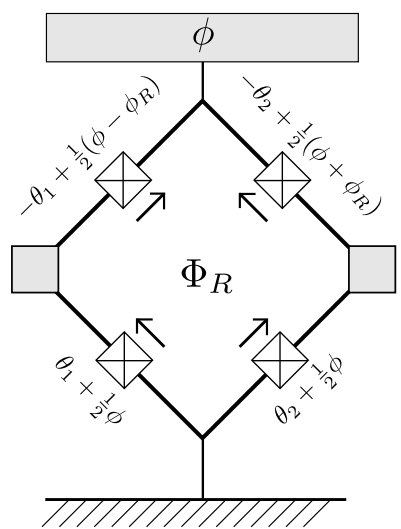

FIG. 2. (a) Circuit of the Josephson rhombus consisting of a loop interrupted by four Josephson junctions with Josephson energies $E_{J_{\diamond}}$ and capacitances $C_{\diamond}$. When the loop is threaded by a flux of $\Phi_{R}=\Phi_{0} / 2$, the transport of single Cooper-pairs through the device is suppressed by destructive interference between the tunneling events through the left and the right arm of the rhombus, rendering the rhombus eigenenergies $\pi$-periodic in the fixed phase difference $\phi$ between the top island and the ground superconductor. (b) Choice of the gauge-invariant phase differences across the links with the arrows indicating their orientation. The phases $\theta_{j}$ are conjugate to the charges $n_{j}=-i \partial / \partial \theta_{j}$ of the superconducting islands (gray squares) in the two arms of the rhombus. The phases add up to the reduced flux $\phi_{R}=2 \pi \Phi_{R} / \Phi_{0}$.

where $\alpha$ is a free parameter. Note that the supercharge $Q$ is Hermitian since the addition/removal $e^{ \pm i \phi}$ of a Cooperpair anticommutes with the Cooper-pair parity $(-1)^{n}$ of the island. We then find that with $\alpha=\sqrt{E_{J 2} / 2 E_{C_{\Sigma}}}$ and up to irrelevant constants, the supercharge $Q$ squares to the Hamiltonian Eq. (1) at the point $n_{g}=0$ and

$$
E_{J 1}=\sqrt{8 E_{J 2} E_{C_{\Sigma}}}
$$

where the system Eq. (1) is supersymmetric. The exotic feature of the supersymmetry to note here is the preservation of the degeneracy of the energy levels all the way from the charge qubit regime with $\alpha \ll 1$ to the transmon regime $\alpha \gg 1$ as long as (3) is fulfilled. The "hidden" character of this degeneracy is underlined by the highly nonlocal form of the supercharge (2).

From the above, we see that an (effective) Josephson junction with a $\pi$-periodic Josephson energy of the form $-E_{J 2} \cos (2 \phi)$ is crucial for supersymmetry. Such a circuit element is provided by the Josephson rhombus shown in Fig. 2(a). ${ }^{17}$ It is a two arm Cooper-pair interferometer connecting the superconducting island to the ground in which single Cooper-pairs tunneling through the left and right arm of the rhombus interfere destructively due to a magnetic flux $\Phi_{R}=\Phi_{0} / 2$. Each arm contains two Josephson junctions connected in series with Josephson energy $E_{C \diamond}$ and capacitance $C_{\diamond}$. We show that in the charging regime $\eta=E_{J \diamond} / 2 E_{C \diamond} \lesssim 1$, the ground state energy of the rhombus is well approximated 
$(a)$

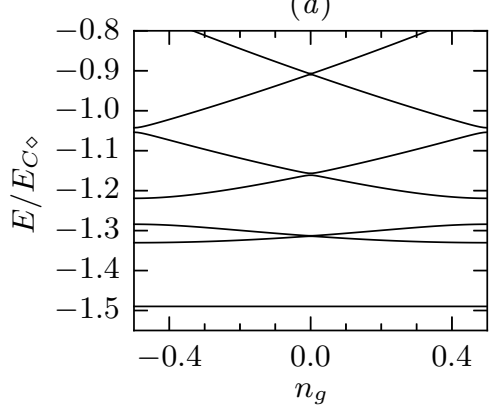

(b)

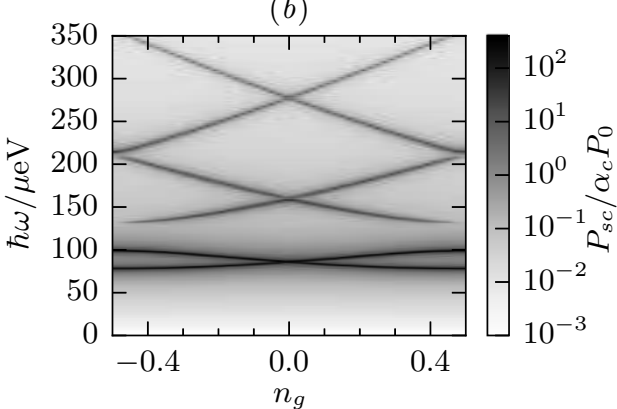

$(c)$

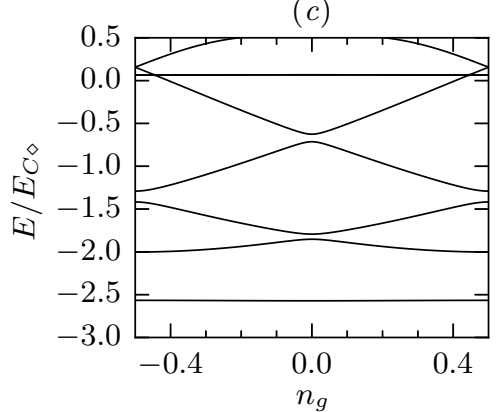

FIG. 3. Energy spectra of the full Hamiltonian Eq. (4) as a function of the dimensionless offset charge $n_{g}=C_{g} V_{g} / 2 e$ for $\alpha=2, \eta=0.7$ in $(a)$ and $\alpha=1, \eta=1$ in $(c)$. For a given $\eta=E_{J \diamond} / 2 E_{C \diamond}$, we numerically determine the strength $E_{J 2}$ of the $\pi$-periodic component of the ground state energy $\epsilon_{0}(\phi)$ of the rhombus. The value of the charging energy is then obtained from the relation $E_{C_{\Sigma}}=E_{J 2} / 2 \alpha^{2}$. The strength of the Josephson coupling $E_{J 1}$ is fixed at a value $4 E_{C_{\Sigma}} \alpha$ which corresponds to the supersymmetric point, cf. (3). It can be seen in (a) that all the excited levels cross at $n_{g}=0$, confirming the validity of the effective supersymmetric model (1) beyond the perturbative regime $\eta \ll 1$. As shown in (c) the former supersymmetric level crossings at $n_{g}=0$ turn into avoided crossings at a slightly elevated $\eta$. This signals the breakdown of the rhombus/island decoupling and thus restricts the mapping of the full Hamiltonian to the supersymmetric model. Additionally, the first excited rhombus level can be seen as a horizontal line around $E / E_{C \diamond}=0$. (b) Plot of the power loss $P_{\mathrm{sc}}$ at frequency $\omega$ of the transmission line coupled to the system with parameters given in $(a)$ which corresponds to the experimental parameters $\left(C_{1}+C_{g}\right) / C_{\diamond}=80, E_{J 1}=0.1 E_{C \diamond}$, and $E_{C \diamond}=500 \mu \mathrm{eV}$, cf. (6). The power loss is measured in units of the injected intensity $P_{0}$ and a dimensionless coupling constant $\alpha_{C}$. Here, we assume that the system once exited relaxes fast with a rate $\Gamma=0.5 \mu \mathrm{eV} / \hbar$ into degrees of freedom different from the transmission line such that the resonance condition is indicated by a dip of size $P_{\mathrm{sc}}$ in the transmitted intensity. Since the ground state is nearly insensitive to changes in $n_{g}$, the power loss can be directly compared to the spectrum shown in $(a)$.

by $\epsilon_{0}(\phi) \approx-E_{J 2} \cos (2 \phi)$, where $\phi$ is the (fixed) phase difference between island and ground. Furthermore, we argue that the weak coupling to the island permits an adiabatic decoupling leading to the effective Hamiltonian (1). To this end, let us denote the Cooper-pair number of the superconducting islands in the left and right arm by $n_{1}, n_{2}$ and choose the gauge-invariant phase differences across the junctions as indicated in Fig. 2(b). Taking into account the additional flux $\Phi_{J R}=-\Phi_{0} / 4$ from Fig. 1, the Hamiltonian of the full system assumes the form

$$
H=4 E_{C_{\Sigma}}\left(n-n_{g}\right)^{2}-E_{J 1} \cos \phi+H_{\diamond},
$$

which corresponds to the effective model Eq. (1) with $-E_{J 2} \cos (2 \phi)$ replaced by the rhombus Hamiltonian

$$
H_{\diamond}=2 E_{C \diamond}\left(n_{1}^{2}+n_{2}^{2}\right)+V_{\diamond}\left(\theta_{1}, \theta_{2}, \phi\right) .
$$

Here, $E_{C \diamond}=e^{2} / 2 C_{\diamond}$ is a charging energy, the phases $\theta_{1}$, $\theta_{2}$ are conjugate to $n_{1}=-i \partial / \partial \theta_{1}, n_{2}=-i \partial / \partial \theta_{2}$, and the potential $V_{\diamond}$ reads

$$
V_{\diamond}=-E_{J \diamond} \sum_{j=1,2}\left[\cos \left(\frac{\phi}{2}+\theta_{j}\right)+\cos \left(\frac{\phi}{2}+(-1)^{j} \frac{\phi_{R}}{2}-\theta_{j}\right)\right],
$$

where $\phi_{R}=2 \pi \Phi_{R} / \Phi_{0}$ is the reduced flux. For fixed $\phi$, the Hamiltonian $H_{\diamond}$ possesses instantaneous eigenstates $|n ; \phi\rangle$ with eigenvalues $\epsilon_{n}(\phi)$. The destructive interference of single Cooper-pair tunneling is expressed by the fact that exchanging the two tunneling paths and advancing $\phi$ by $\pi$ is a symmetry of the system,,$^{28}$ demanding $\pi$-periodicity of $\epsilon_{n}(\phi)$. At half a flux quantum, time-reversal is an additional symmetry demanding an even $\epsilon_{n}(\phi)$. Consequently, the ground state energy $\epsilon_{0}(\phi)$ must be of the form $\epsilon_{0}(\phi)=-\sum_{n} E_{J 2 n} \cos (2 n \phi)$. By perturbation theory in $\eta$, we find that the desired $\pi$ periodic component $E_{J 2} / E_{C \diamond}=7 \eta^{4} / 4$ dominates, with $E_{J 4} / E_{C \diamond}=-68687 \eta^{8} / 36864$ and $E_{J 2 m} / E_{C \diamond} \propto \eta^{4 m}$. In the following, we are interested in the regime $\eta \lesssim 1$ and thus we determine $E_{J 2}, E_{J 4}$ numerically from $\epsilon_{0}(\phi)$ whenever needed. We find that $E_{J 2}$ stays at least an order of magnitude larger than $E_{J 4}$ up to $\eta \approx 1$. Since the above form of the rhombus energies is due to symmetry, differing Josephson couplings $E_{J \diamond, L / R}=E_{J \diamond}(1 \pm \delta / 2)$ in the left and right rhombus arm will in general induce a finite $2 \pi$-periodic Josephson coupling whose strength scales perturbatively as $4 \eta^{2} \delta E_{C \diamond}$. Comparison with $E_{J 2}$ yields that the effects of asymmetry are negligible for $\eta^{2} \gg \delta$.

Projecting the Hamiltonian Eq. (4) onto the instantaneous rhombus ground state $|0 ; \phi\rangle$ and using $\epsilon_{0}(\phi) \approx$ $-E_{J 2} \cos (2 \phi)$ leads by standard methods ${ }^{29}$ to the effective Hamiltonian $H_{\mathrm{ad}}=H_{\text {eff }}+4 E_{C_{\Sigma}} \sum_{n>0}\left|A_{n 0}\right|^{2}$, where $A_{n m}=i\left\langle n ; \phi\left|\partial_{\phi}\right| m ; \phi\right\rangle$ is the induced vector potential describing the nonadiabatic corrections. In deriving $H_{\mathrm{ad}}$, we have used that the term $A_{00}$ vanishes since the states $|m ; \phi\rangle$ can be chosen real. Due to timereversal and rhombus symmetry, $\left|A_{n 0}\right|$ is even in $\phi$ and thus does not couple the supersymmetric partners at $n_{g}=0$. Since the gap to the next pair of supersymmetric states is at least of order $E_{C_{\Sigma}}$, the effects of $\left|A_{n 0}\right|^{2}=$ $\left|\left\langle n ; \phi\left|\partial_{\phi} H_{\diamond}\right| 0 ; \phi\right\rangle\right|^{2} /\left[\epsilon_{n}(\phi)-\epsilon_{0}(\phi)\right]^{2} \propto \eta^{2}$ are negligible for 
$\eta \ll 1$. The coupling to the excited rhombus levels that we do not take into account in the projected Hamiltonian $H_{\text {ad }}$ is suppressed by even higher orders in $\eta$.

The supersymmetry becomes trivial for $\alpha \rightarrow 0$ where one recovers the supersymmetry of the free particle. ${ }^{14} \mathrm{We}$ therefore aim for the most interesting regime of $\alpha \approx 1$, where all the terms in the Hamiltonian (1) are of the same order. While the adiabatic decoupling of the rhombus is most robust for large scale separation $\eta \ll 1$ between the rhombus and the island, our perturbative results for $E_{J 2}$ show that this also implies $\alpha \propto \eta^{2} \ll 1$. The regime $\alpha \approx 1$ thus requires moderately large $\eta$ for which we numerically show that the adiabatic decoupling still works. Figure 3(a) shows the numerical results for the spectrum of the full Hamiltonian as a function of the offset charge $n_{g}$ for $\alpha=2$ and $\eta=0.7$. We highlight that all excited levels, including the levels higher in energy not visible in Fig. 3(a), become doubly degenerate as $n_{g}$ approaches zero. This degeneracy of all excited states in complete absence of selection rules gives a clear signature of supersymmetry. Figure 3(c) shows the high sensitivity of the supersymmetry to the choice of $\eta$. For $\alpha=1$ and $\eta=1$, supersymmetry at $n_{g}=0$ is clearly destroyed by nonadiabatic corrections in the Hamiltonian $H_{\text {ad }}$. Moreover, the first excited level of the rhombus showing up as a horizontal line in the upper region of Fig. 3(c) is visible.

The spectrum of the system can be read out with the help of the transmission line coupled to the island by injecting a voltage $V(t)=V_{g}+V_{\omega} \cos (\omega t)$ with the AC amplitude $V_{\omega}$ at the frequency $\omega$. For a transmission line characterized by the admittance $Y_{\mathrm{tl}}$, this corresponds to an average injected intensity $P_{0}=\frac{1}{2} Y_{\mathrm{t} 1} V_{\omega}^{2}$. According to the Hamiltonian (4), the $\mathrm{AC}$ voltage drives transitions in the system through the coupling $H_{C}=g_{C} V(t) n$ with $g_{C}=2 e C_{g} / C_{\Sigma}$. Due to the coupling to the charge $n$, the driving is most effective for $\alpha \gtrsim 1$. We assume that the system relaxes dominantly into channels different from the transmission line with a rate $\Gamma \gg \Gamma_{\text {abs }}$, where $\Gamma_{\text {abs }}$ is the rate of photon absorption. Measuring in transmission, the absorption of photons is then signaled by a reduced transmitted intensity $P_{t}$ with respect to the incoming intensity $P_{0}$. With $\Gamma \gg \Gamma_{\text {abs }}$, photons of energy $\hbar \omega$ exclusively drive transitions from the ground state to excited states and the scattered intensity $P_{\mathrm{sc}}=P_{0}-P_{t}$ follows as $P_{\mathrm{sc}}=\hbar \omega \Gamma_{\mathrm{abs}}$. For the calculation of $\Gamma_{\mathrm{abs}}$, we employ Fermi's golden rule. We find

$$
P_{\mathrm{sc}}=4 \alpha_{C} P_{0} \sum_{n>0} \frac{\hbar^{2} \omega \Gamma\left|\left\langle n\left|i \partial_{\phi}\right| 0\right\rangle\right|^{2}}{\left(E_{n}-E_{0}-\hbar \omega\right)^{2}+\hbar^{2} \Gamma^{2}},
$$

denoting the eigenstates to energy $E_{n}$ of the full Hamiltonian (4) by $|n\rangle$; here, the dimensionless constant $\alpha_{C}$ is given by $\alpha_{C}=g_{C}^{2} / \hbar Y_{\mathrm{tl}}=2 \pi C_{g}^{2} / C_{\Sigma}^{2} Z_{0} Y_{\mathrm{tl}}$ with the superconducting impedance quantum $Z_{0}=h / 4 e^{2} \approx 1 \mathrm{k} \Omega$. The condition $\Gamma_{\text {abs }} \ll \Gamma$ translates into $g_{C} V_{\omega} \ll \hbar \omega$. The validity of Fermi's golden rule for the rate calculation on the other hand demands $\alpha_{C} \omega \ll \Gamma$.

The system with $\alpha=2$ presented in Fig. 3(a) corresponds to the experimental parameters $\eta=0.7,\left(C_{1}+\right.$
$\left.C_{g}\right) / C_{\diamond}=80$ and $E_{J 1}=0.1 E_{C \diamond}$. In Fig. 3(b), we show the numerical results for the scattered intensity $P_{\mathrm{sc}}$ as a function of the offset charge $n_{g}$ and the radiation frequency $\omega$ with the system parameters stated above. We assume the experimental scale $E_{C \diamond}=500 \mu \mathrm{eV}$. As visible from Fig. 3(a), the ground state is almost insensitive to changes of $n_{g}$ at $\alpha=2$ such that the scattered intensity corresponds directly to the energy spectrum of the system. The scattered intensity is strongest for the first degenerate pair of levels which reflects the fact that states higher in energy show a behavior closer to the charging regime than the low-energy states. Note that the whole spectrum and the level crossings of all excited states at the supersymmetric point (3) with $n_{g}=0$ can be clearly observed.

As a last point, let us comment on the susceptibility to imperfections in design. The above analysis was based on the rhombus symmetry which is violated both by stray offset charges or parameter variations within the offset arms as parametrized by $\delta$. As we explain in more detail in the Appendix, the susceptibility to stray offset charges can in practice be reduced by adding inductive shunts to the ground within the rhombus arms. ${ }^{19,21}$ For the system parameters chosen above, numerical checks show that symmetry violations $\delta$ are tolerable up to a few percent. The most demanding experimental requirements are thus the reproducible parameter of the rhombus junctions. In contrast, deviations in $C_{1} / C_{\diamond}$ can be accounted for by the Josephson coupling $E_{J 1}$ which can be tuned easily.

To conclude, we have shown that a quantummechanical supersymmetry arises in a Cooper-pair box when it is shunted by a Josephson rhombus operated in the charging regime, where it is weakly coupled to the Cooper-pair box and generates an effective $\pi$-periodic Josephson coupling of the form $\cos (2 \phi)$. The supersymmetry is nontrivial since there are no selection rules and an analytic solution is impossible, but yet, it guarantees an exact degeneracy of all excited levels. We have shown that the supersymmetry can be detected through microwave spectroscopy and tuning in and out of the supersymmetric point is easily possible by tuning the gate voltage. We have proposed realistic device parameters, paving the way for an experimental exploration of the exotic degeneracies brought by supersymmetries in the near future.

The authors acknowledge financial support from the Alexander von Humboldt Foundation and the Deutsche Forschungsgemeinschaft under Grant No. HA 7084/2-1 as well as useful discussions with Christoph Ohm.

\section{Appendix A: Device with inductive shunts}

The simple rhombus design that we have presented in the main text for clarity of the discussion suffers from the fact that the charge configuration on the capacitors of the central islands in the rhombus is strongly 


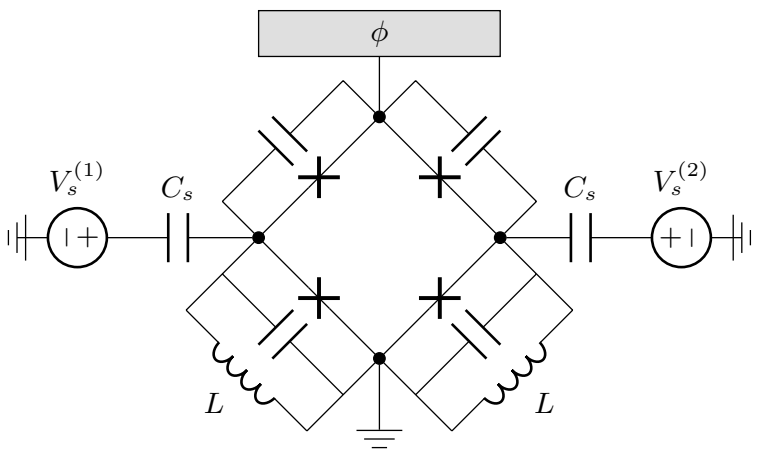

FIG. 4. Rhombus design as proposed theoretically in Ref. 19 which includes inductances $L$ that shunt the islands in the rhombus arms and reduce the susceptibility to stray offset charges coupling capacitively to the islands. Modeling the offset charges through fluctuating voltage sources that charge capacitors $C_{s}$ coupled to the islands yields the relation $n_{s}^{(j)}=$ $C_{s} V_{s}^{(j)} / 2 e$ for the stray offset charge $n_{s}^{(j)}$. Apart from the change in rhombus design, the proposed setup remains the same as in Fig. 1. Importantly, it is sufficient to add inductive shunts only to the lower part of the rhombus arms such that no inductive coupling to the main island is generated.

susceptible to fluctuations of stray offset charges that couple capacitively to the islands. They can be modeled, e.g., through voltage sources $V_{s}^{(1) /(2)}$ that charge capacitors $C_{s}$ coupled to the rhombus islands, yielding $n_{s}^{(1) /(2)}=C_{s} V_{s}^{(1) /(2)} / 2 e$ for the stray offset charge $n_{s}^{(1) /(2)}$; see Fig. 4. These stray offset charges are not controlled and fluctuate in general independently, destroying the symmetry of the rhombus arms and lifting the $\pi$-periodicity of the rhombus. As was argued theoretically ${ }^{19}$ and tested experimentally, ${ }^{21}$ this can be remedied by adding inductive shunts of strength $L$ to the lower parts of the rhombus arms. In the classical case and ignoring the Josephson junctions, adding inductive shunts to the ground in the central islands of the rhombus reduces this susceptibility by a factor of $\omega^{2} / \omega_{L C}^{2}$, where $\omega$ is the frequency of offset charge fluctuations and $\omega_{L C}=1 / \sqrt{2 L C_{\diamond}}=\sqrt{4 E_{C \diamond} E_{L}} / \hbar$ is the plasma frequency of charge oscillations in the resulting LC resonator with the inductive energy $E_{L}=\left(\Phi_{0} / 2 \pi\right)^{2} / L$. As was shown in Ref. 30, this property carries over to the quantum case. As a consequence of the inductive shunts, the rhombus does not couple any more directly to $n_{s}^{(1) /(2)}$ but only to $\dot{n}_{s}^{(1) /(2)}$. The associated noise power changes from the $1 / \omega$ form typical for fluctuations of $n_{s}^{(1) /(2)} 31$ to a much more benign noise power proportional to $\omega$.

As we have discussed above, the inclusion of inductive shunts reduces the sensitivity to offset charge fluctuations, but it should of course also preserve the behavior of the rhombus as an effective $\cos (2 \phi)$ element. This means that in our setup, we cannot make $E_{L}$ arbitrarily large since our treatment required that both the band indices and the Bloch momenta $q_{i}$ associated with the translational symmetry in the variables $\theta_{i}$ in absence of inductive shunts remain good variables. This means that $E_{L}$ should be a weak perturbation with $E_{L} \ll E_{J \diamond}, E_{C \diamond}$. We can therefore follow the ideas of Refs. 30 and 32 and transform to a basis of Bloch waves. Solving the $2 \pi$ periodic part of the rhombus perturbatively in $\eta$ and projecting on the lowest band of the rhombus yields a Hamiltonian of the form

$$
\begin{aligned}
H_{\diamond}^{\prime}= & \sum_{j=1,2}\left\{\frac{E_{L}}{2}\left(i \frac{d}{d q_{j}}\right)^{2}+f\left(q_{j}\right)\right. \\
& \left.+g\left(q_{j}\right) \cos (2 \phi)+(-1)^{j} h\left(q_{j}\right) \sin (\phi)\right\},
\end{aligned}
$$

with periodic functions $f, g, h$ with period 1 . The absence of a coupling between $q_{1}$ and $q_{2}$ reflects the fact that the phases $\theta_{j}$ are not coupled by the rhombus Hamiltonian. The presence of a coupling to $\cos (2 \phi)[\sin (\phi)]$ that is even [odd] under the exchange $q_{1} \leftrightarrow q_{2}$ reflects the symmetry under exchange of the rhombus arms and simultaneous advance of $\phi$ by $\pi$ that we discussed in the main text. The additional time-reversal symmetry at half a flux quantum forbids a coupling to $\cos (\phi)$ or $\sin (2 \phi)$ and requires that the functions $f, g, h$ possess quadratic expansions around $q=0$ which we find to be of the form

$$
\begin{aligned}
& f(q) / E_{C \diamond}=2 q^{2}\left(1+\mathcal{O}\left(\eta^{2}, q^{2}\right)\right)+\mathcal{O}\left(\eta^{4}\right), \\
& g(q) / E_{C \diamond}=-\frac{7}{8} \eta^{4}\left(1+\frac{111}{7} q^{2}+\mathcal{O}\left(\eta^{2}, q^{4}\right)\right), \\
& h(q) / E_{C \diamond}=-8 \eta^{2} q^{2}\left(1+\mathcal{O}\left(\eta^{2}, q^{2}\right)\right) .
\end{aligned}
$$

Obviously, for $q_{1}=q_{2}=0$ and $E_{L}=0$, the Hamiltonian $H_{\diamond}^{\prime}$ reproduces the ground state energy $\epsilon_{0}(\phi)$ that was given in the main text. For finite $E_{L}$, the former rhombus eigenstates with sharp Bloch momenta $q_{1}, q_{2}$ are replaced by eigenstates of the Hamiltonian (A1). Assuming a symmetric state of the rhombus arms, the ground state energy $\epsilon_{0}^{\prime}(\phi)$ of $H_{\diamond}^{\prime}$ can still be expanded in the form $\epsilon_{0}^{\prime}(\phi)=-\sum_{n} E_{J 2 n}^{\prime} \cos (2 n \phi)$. Importantly, $E_{L} \ll E_{C \diamond}$ implies $q^{2} \sim \sqrt{E_{L} / 4 E_{C \diamond}} \ll 1$ which guarantees that the term $E_{J 2}^{\prime}$ remains dominant in the expansion of $\epsilon_{0}^{\prime}(\phi)$. Numerical checks show that this property persists beyond the perturbative regime in $\eta$ and remains valid also for $\eta=0.7$. The inductive shunts thus manage to reduce the susceptibility to charge noise without spoiling the generation of an effective $\cos (2 \phi)$ potential by the rhombus.

Finally, let us comment on the adiabatic decoupling of the island and the rhombus for finite $E_{L}$. The level spacing of the eigenstates of the Hamiltonian (A1) is of the order of the plasma energy $\hbar \omega_{L C}$. In view of the adiabatic decoupling, it is desirable to have $\hbar \omega_{L C} \gtrsim E_{\mathrm{SUSY}}$, i.e., $E_{L} \gtrsim E_{\mathrm{SUSY}}^{2} / E_{C \diamond}$, where $E_{\mathrm{SUSY}}$ is the energy scale of the rhombus. We note that for $\alpha=2$ and $\eta=0.7$, we have $E_{\mathrm{SUSY}} \approx E_{J 1}=E_{C \diamond} / 10$ such that choosing $E_{L} \gtrsim E_{\mathrm{SUSY}}^{2} / E_{C \diamond}=E_{C \diamond} / 100$ and $E_{L} \ll E_{C \diamond}, E_{J \diamond}$ is easily possible. Following Ref. 21, such an inductance can 
in practice be implemented through a chain of Josephson junctions.

1 A. van Oudenaarden and J. E. Mooij, Phys. Rev. Lett. 76, 4947 (1996).

2 M. Neeley, M. Ansmann, R. C. Bialczak, M. Hofheinz, E. Lucero, A. D. O'Connell, D. Sank, H. Wang, J. Wenner, A. N. Cleland, M. R. Geller, and J. M. Martinis, Science 325, 722 (2009).

3 J. Li, M. P. Silveri, K. S. Kumar, J. M. Pirkkalainen, A. Vepsalainen, W. C. Chien, J. Tuorila, M. A. Sillanpaa, P. J. Hakonen, E. V. Thuneberg, and G. S. Paraoanu, Nat. Commun. 4, 1420 (2013).

${ }^{4}$ P. D. Nation, M. P. Blencowe, A. J. Rimberg, and E. Buks, Phys. Rev. Lett. 103, 087004 (2009).

5 B. Douçot, L. B. Ioffe, and J. Vidal, Phys. Rev. B. 69, 214501 (2004).

6 D. Marcos, P. Rabl, E. Rico, and P. Zoller, Phys. Rev. Lett. 111, 110504 (2013).

7 B. van Heck, E. Cobanera, J. Ulrich, and F. Hassler, Phys. Rev. B 89, 165416 (2014).

8 I. M. Georgescu, S. Ashhab, and F. Nori, Rev. Mod. Phys. 86, 153 (2014).

9 G. S. Paraoanu, J. Low. Temp. Phys. 175, 633 (2014).

10 A. Messiah, Quantum Mechanics, vol. II (North-Holland, Amsterdam, 1958).

11 E. Witten, Nucl. Phys. B 188 (3), 513 (1981).

12 J. Ulrich, I. Adagideli, D. Schuricht, and F. Hassler, Phys. Rev. B 90, 075408 (2014).

13 I. Aitchison, Supersymmetry in Particle Physics: An Elementary Introduction (Cambridge University Press, Cambridge, UK, 2007).

14 A. R. P. Rau, J. Phys. A 37, 10421 (2004).

15 V. A. Andreev and P. B. Lerner, Phys. Lett. A 134, 507 (1989).
16 M. S. Plyushchay, Mod. Phys. Lett. A 11, 397 (1996).

17 B. Douçot and J. Vidal, Phys. Rev. Lett. 88, 227005 (2002).

18 L. B. Ioffe, M. V. Feigel'man, A. Ioselevich, D. Ivanov, M. Troyer, and G. Blatter, Nature (London) 415, 503 (2002).

19 B. Douçot and L. Ioffe, Rep. Prog. Phys. 75, 072001 (2012).

20 S. Gladchenko, D. Olaya, E. Dupont-Ferrier, B. Douçot, L. B. Ioffe, and M. E. Gershenson, Nat. Phys. 5, 48 (2009).

21 M. T. Bell, J. Paramanandam, L. B. Ioffe, and M. E. Gershenson, Phys. Rev. Lett. 112, 167001 (2014).

22 L. B. Ioffe and M. V. Feigel'man, Phys. Rev. B 66, 224503 (2002).

23 I. V. Protopopov and M. V. Feigel'man, Phys. Rev. B 70, 184519 (2004).

24 V. Bouchiat, D. Vion, P. Joyez, D. Esteve, and M. H. Devoret, Phys. Scr. T76, 165 (1998).

25 Y. Nakamura, Y. A. Pashkin, and J. S. Tsai, Nature (London) 398, 786 (1999).

${ }^{26}$ F. Cooper, A. Khare, and U. Sukhatme, Supersymmetry in Quantum Mechanics (World Scientific, Singapore, 2001).

27 M. Combescure, F. Gieres, and M. Kibler, J. Phys. A 37, 10385 (2004).

28 This corresponds to the transformation $\theta_{1} \mapsto \theta_{2}-\pi / 2$, $\theta_{2} \mapsto \theta_{1}-\pi / 2$, and $\phi \mapsto \phi+\pi$.

29 A. Böhm, H. Koizumi, Q. Niu, J. Zwanziger, and A. Mostafazadeh, The Geometric Phase in Quantum Systems (Springer, Berlin, 2003).

30 J. Koch, V. Manucharyan, M. H. Devoret, and L. I. Glazman, Phys. Rev. Lett. 103, 217004 (2009).

31 O. Astafiev, Y. A. Pashkin, Y. Nakamura, T. Yamamoto, and J. S. Tsai, Phys. Rev. Lett. 93, 267007 (2004).

32 G. Schön and A. D. Zaikin, Phys. Rep. 198, 237 (1990). 